\title{
Vitreous and Optic Nerve Infiltration of an Adrenal B Lymphoma
}

\author{
Lucía Galletero Pandelo $^{a} \quad$ Cristina Rodríguez Vidala Joseba Artaraz ${ }^{a}$ \\ Nerea Martínez-Alday ${ }^{a} \quad$ Alex Fonollosa ${ }^{a}$ b \\ aDepartment of Ophthalmology, BioCruces Bizkaia Health Research Institute, Cruces \\ University Hospital, University of the Basque Country, Baracaldo, Spain; ${ }^{\text {bBilbao }}$ \\ Ophthalmological Institute, Bilbo, Spain
}

\section{Keywords}

Lymphoma $\cdot$ Metastases $\cdot$ Vitritis · Optic disc edema $\cdot$ Methotrexate

\begin{abstract}
Aim: This study aimed to report a case of vitreous and optic nerve infiltration of a primary adrenal B lymphoma. Case Report: An 81-year-old patient consulted for decrease in vision in the left eye and pain with ocular movements during the last week. The patient was being treated with chemotherapy for an adrenal gland diffuse large B-cell lymphoma. On examination, vitritis was seen in both eyes and optic disc edema in his left eye. After an extensive study based on orbital and brain magnetic resonance imaging and vitreous cytology, an ocular infiltration by systemic B lymphoma was confirmed. A treatment based on intravitreal methotrexate was carried out, achieving the regression of the vitreous infiltration and resolution of the optic disc edema. Systemic B lymphoma metastasizing ocular structures is extremely infrequent. The manifestations may mimic an inflammatory disease. Ophthalmologists should be aware of these manifestations and consider among the masquerade syndromes.
\end{abstract}

(C) 2021 The Author(s).

Published by S. Karger AG, Basel

\section{Introduction}

Intraocular lymphoma (IOL) is relatively uncommon, representing $<1 \%$ of all intraocular tumors. Primary intraocular B-cell lymphoma is the most common lymphoma in the eye $[1,2]$; nevertheless, ocular involvement in the context of metastatic lymphoma accounts for $17 \%$ of IOLs [1] and is found in $6.7-23.3 \%$ of patients with lymphoma [3]. 


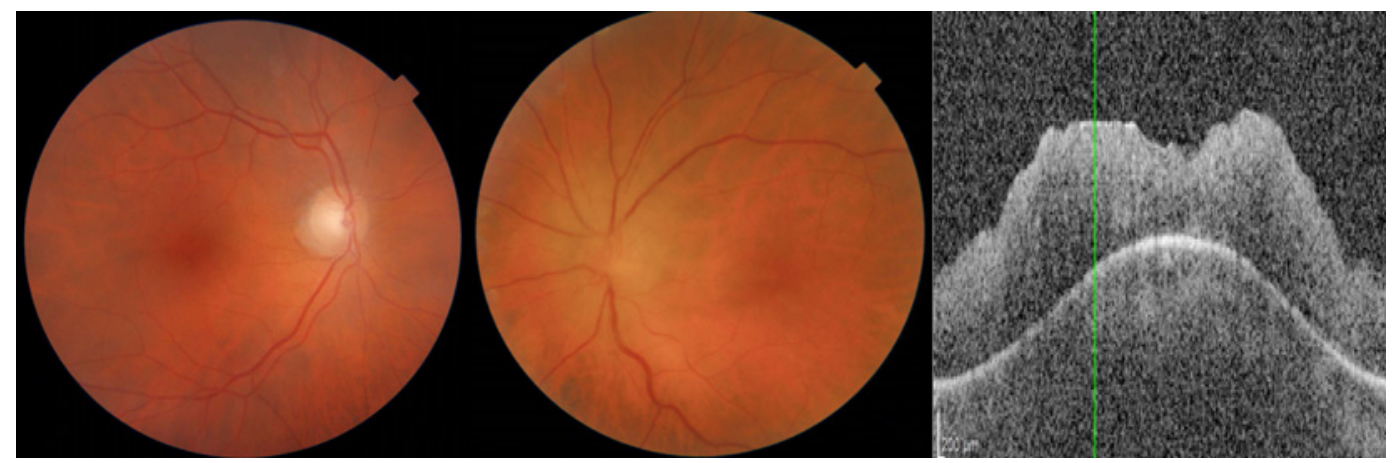

Fig. 1. Initial fundus examination. Left: normal posterior pole of the right eye. Middle: edematous papilla in the left eye and vitreous opacities. Right: OCT image showing optic disc edema.

Ocular involvement has been associated with a reduced median overall survival. Alqurashi and Alqahtani [4] reported a reduction in median overall survival from 12.5 months to 4 months. However, if such lesions are detected early enough, they can be treated effectively to improve vision and to maintain the quality of the patient's life.

Even more rare are intraocular manifestations of metastatic adrenal lymphoma, with only 1 case of adrenal T-cell lymphoma and another of non-Hodgkin large-cell lymphoma described $[2,5]$. We report the case of a patient with an adrenal gland large B-cell lymphoma who presented metastatic disease in the vitreous and the optic nerve, a complication that has not previously been described.

\section{Case History}

An 81-year-old man, known case of adrenal B lymphoma, consulted for decreased vision in the left eye and orbital pain with ocular movements of 7-day duration. At the time of the examination, the patient had a visual acuity of 20/40 in the right eye, as opposed to light perception vision in the left eye. Anterior segment biomicroscopy did not show abnormalities. Intraocular pressure was $12 \mathrm{~mm} \mathrm{Hg}$ in both eyes. Pupils were both reactive to light, with no relative afferent pupillary defect. Funduscopy showed vitreous cells in both eyes, with more abundance in the left eye, and the left optic disc was edematous. Optical coherence tomography was done which confirmed optic nerve edema (shown in Fig. 1).

His medical history was relevant for a large B-cell adrenal lymphoma diagnosed 3 months earlier, for which the patient was receiving treatment consisting of cycles of chemotherapy (R-CHOP protocol: rituximab, cyclophosphamide, adriamycin, vincristine, and prednisolone). A positron emission tomography performed at the time of the adrenal lymphoma diagnosis revealed cervical and retroperitoneal adenopathies and spleen involvement, thus making it a tumor in the IVB stage according to the Ann Arbor classification. The initial ophthalmological presentation started after receiving 6 cycles of systemic chemotherapy. At that moment, the patient did not show any systemic manifestations.

An orbital and brain MRI with gadolinium was performed revealing evidence of thickening and enhancement of the left optic disc (shown in Fig. 2), suggestive of infiltrative process. There were no radiological signs suggestive of neoplastic meningitis (NM). The examination of cerebral spinal fluid including cytological evaluation for malignant cells was normal.

A clinical picture of bilateral vitritis and optic disc edema confirmed by OCT in an elderly man with a recent diagnosis of large B-cell adrenal lymphoma and thickening and enhancement 
Fig. 2. Axial T2 flair MR image revealing hypersignal and enhancement of the left optic nerve.

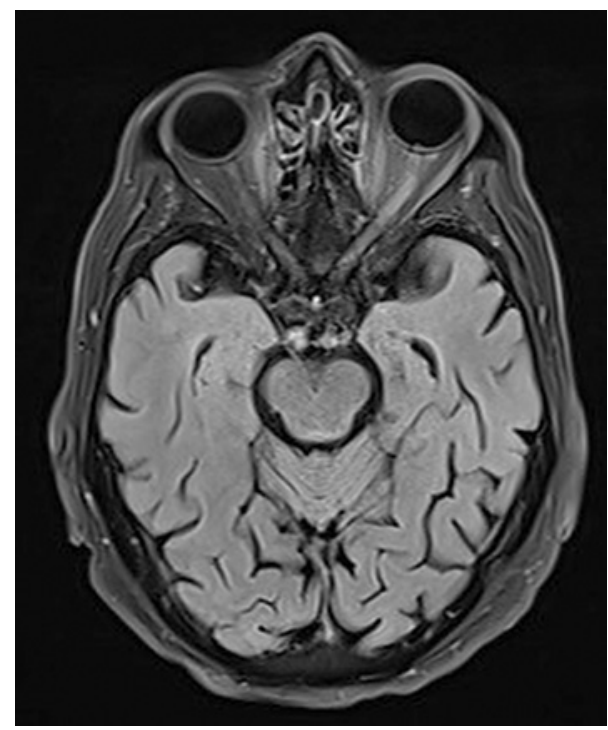

of the left optic disc in MRI suggestive of infiltrative process restricted greatly the differential diagnosis. Although some other etiologies could have been taken into account (basically other oncohematologic processes, meningiomas, gliomas, or sarcoidosis), an intraocularinvolvement of the primary neoplasm was the most likely; hence, at first, we did not consider any ancillary tests apart from confirming the intraocular infiltration by means of a diagnostic vitrectomy, which was made in the left eye. Cytology revealed the presence of atypical large lymphocytes. Flow cytometry showed $88.8 \%$ of lymphocytes B CD19+ CD20+ CD22+ sLambda+, so intraocular asymmetric bilateral infiltration of lymphoma was confirmed. Consequently, a treatment based on intravitreal methotrexate (MTX) and salvage chemotherapy with temozolomide were carried out. It was decided that MTX was to be given just in the left eye because it was the most severely affected.

The patient received a total of 2 intravitreal injections of MTX ( $400 \mu \mathrm{g} / 0.1 \mathrm{~mL})$ in the left eye 4 weeks apart. At 4 weeks of the last injection, visual acuity continued as light perception in the left eye. However, funduscopy showed a clear vitreous cavity in both eyes, and the optic disc edema had resolved (shown in Fig. 3). Autofluorescence and SD-OCT performed at that moment showed multiple hyperautofluorescent spots and hyperreflective globular lesions at the level of the retinal pigment epithelium, suggestive of intraocular infiltration of lymphoma. Seven weeks after the last intravitreal injection, the patient presented a swollen optic disc in the left eye again (shown in Fig. 3). At that moment, the patient's condition had deteriorated due to brain metastases, and he passed away shortly thereafter.

\section{Discussion}

In this case report, we describe the clinical manifestations and evolution of a patient with a primary adrenal lymphoma (PAL) infiltrating the vitreous and the optic nerve. The patient presented bilateral vitritis and unilateral optic disc edema which responded well to systemic chemotherapy and intravitreal MTX. These lesions recurred after a short follow-up with brain metastases, which subsequently caused the death in our patient.

PAL is extremely infrequent [5-9], with $<200$ cases reported in the English literature [6]. For its diagnosis is necessary to exclude prior history of lymphoma elsewhere, and if there is, adrenal lesions must be unequivocally dominant $[5,6,8]$. PAL is bilateral in about $70 \%$ 


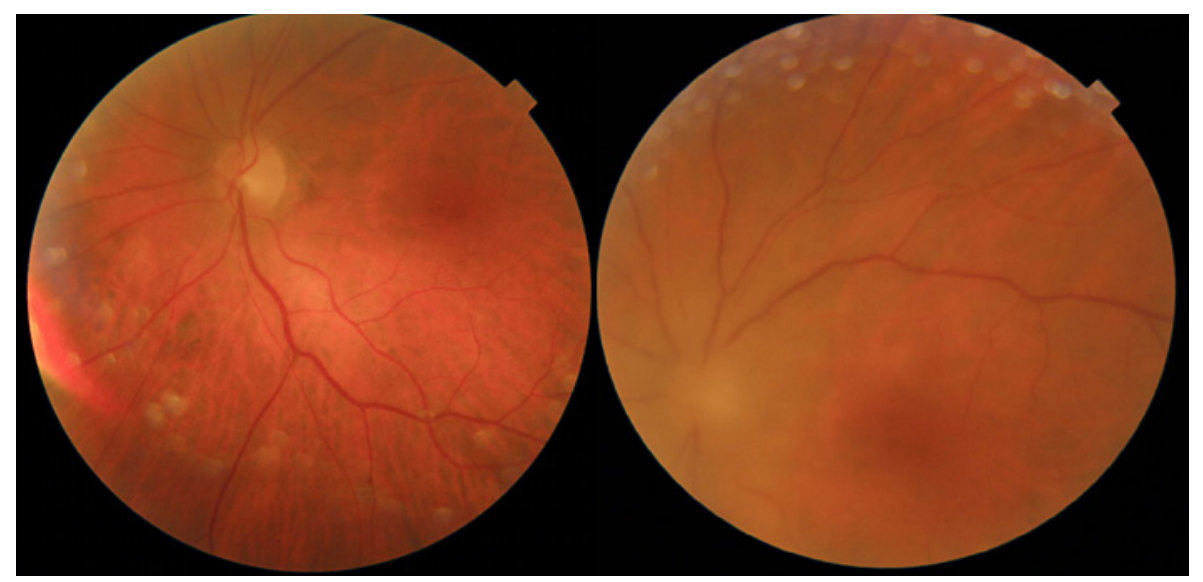

Fig. 3. Left: left eye fundus examination 1 month after intravitreal MTX. Resolution of the optic disc edema is observed. Right: left eye fundus examination 7 weeks after the last intravitreal injection. A swollen optic disc is noted again.

of cases; the most common type described is diffuse large B-cell lymphoma, which comprises more than half of the cases [6]. The prognosis is poor. Rashidi and Fisher [6] reported a 12 -month mortality rate of $80 \%$. The main clinical symptoms are B symptoms (fever, weight loss, and night sweat), pain, and fatigue. Adrenal insufficiency manifested by hyperpigmentation, fatigue, and weight loss occurs in $30-61 \%$ of patients and more often in bilateral disease [6, 9]. However, ocular metastases are rare.

It has been described an elevated LDH in $88 \%$ of cases. When the PAL is unilateral, the frequency of elevated LDH is lower (64\%) [6]. In our case, our patient had a unilateral PAL and normal LDH values. On the other hand, secondary CNS involvement occurs in 2-10\% and confers a poorer prognosis with 12-month survival rates being 8\% [6]. Since the eye is considered a part of the CNS (during embryonic development of the retina and optic nerve extend from the diencephalon), intraocular infiltration of this lymphoma may be considered actually CNS involvement and hence a poor prognosis. In fact, our patient presented brain metastases.

MRI is a preferred neuroradiographic method but is limited with a sensitivity of $48 \%$ detecting NM [10]. In our case, the first MRI showed involvement of the optic nerve without signs of CNS involvement or NM, but since the patient died because of brain metastasis, it cannot be guaranteed that there was no involvement at that time.

Another relevant characteristic is that immune dysfunction has been implicated in the pathogenesis, for example, Epstein-Barr virus positivity is observed in more than half of the cases. However, in our patient, no previous immunodeficiency was observed [6].

Regarding intraocular involvement of this disease, as far as we know, only 1 case has been published previously. Schocket et al. [5] described a 71-year-old man who consulted for decreased visual acuity due to a serious retinal detachment and infiltrative choroidal lesions in his left eye; systemic investigations revealed a PAL without renal or retroperitoneal involvement, so the infiltrative lesions were considered to be choroidal metastases. Chemotherapy resolved both the adrenal lymphoma and the eye disease, and after 7 years of followup, the patient was alive. The ocular involvement of this case was different from ours, showing clinical signs that are usually seen in primary retinal lymphoma or CNS lymphoma. Moreover, life prognosis was also completely different and was probably related to the stage of the disease at initial diagnosis (ocular and isolated adrenal lymphoma in Schocket e al.'s [5] case and ocular, retroperitoneal, and spleen involvement as well as cervical adenopathies in ours).

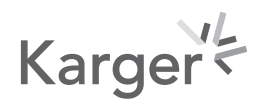


Bedaiwi et al. [2] also reported a case of intraocular manifestations of metastatic T-cell adrenal lymphoma. In this case, the patient presented anterior uveitis with pinkish hypopyion and posterior synechia. Left eye fundus exam showed whitish retinal infiltrate in multiple areas, but neither vitritis nor optic disc edema.

Regarding pathogenesis of metastases, it has been described that they occur via hematogenous spread. Ocular metastasis most frequently occurs in the choroid, which is a richly vascularized tissue, but retinal vessels may also contribute to the dissemination of the tumor into the vitreous and retina $[1,2,11]$. In our case, the lack of lymphatic vessels of the adrenal parenchyma supports this theory [6].

We treated the patient with intravitreal injections of MTX. Most experience of IOL treatment comes from PVRL treatment. In this regard, intravitreal MTX was shown to be effective in small nonrandomized trials [12-15], so we thought it could be a good option in our case. Another local option is rituximab. Small, nonrandomized studies have demonstrated the activity of intravitreal rituximab monotherapy for PVRL $[16,17]$. There is also some evidence that the combination of intravitreal MTX and rituximab may be effective for PVRL [18]. This alternating, combination approach is potentially attractive because it decreases the need for multiple MTX injections with concomitant toxicity. Other options to manage PVRL include external-beam radiotherapy and systemic chemotherapy.

Finally, the diagnosis of vitritis, papillitis, and/or retinal or subretinal infiltrates in older patients should prompt the ophthalmologist to search for vitreoretinal lymphoma. In this clinical scenario, especially in doubtful cases, diagnostic vitrectomy to perform cytology and immunophenotyping by immunochemistry or flow cytometry to demonstrate clonality is essential.

A great proportion of IOLs are primary retinal lymphoma- or CNS-associated lymphoma which is usually bilateral [11]. However, as seen in our case, systemic lymphomas may also metastasize to the eye, and hence it is important to be aware of the possibility of a systemic origin when an IOL is diagnosed.

\section{Acknowledgment}

There have been no substantive contributions to the manuscript.

\section{Statement of Ethics}

This study protocol was reviewed and the need for approval was waived by the Ethics Committee of Cruces University Hospital. Written informed consent was obtained from the patient for publication of this case report and any accompanying images.

\section{Conflict of Interest Statement}

The authors report no conflicts of interest.

\section{Funding Sources}

The authors have no financial disclosure. The authors alone are responsible for the content and preparation of this manuscript.

\section{Karger'}




\section{Author Contributions}

L.G.P. and A.-F. contributed to conceptualization; L.G.P., C.R.V., J.A., and N.M.A. contributed to writing - original draft preparation; L.G.P. and A.F. contributed to writing - review and editing; A.F. contributed to supervision. All authors have read and agreed to the published version of the manuscript.

\section{Data Availability Statement}

Publicly available datasets were used in this study. These can be found in PubMed at http://doi.org/10.4103/iju.IJU_113_18 and http://doi.org/10.7759/cureus.4049 and ScienceDirect at http://doi.org/10.1016/S0002-9394(02)01681-1.

\section{References}

1 Cao X, Shen D, Callanan DG, Mochizuki M, Chan CC. Diagnosis of systemic metastatic retinal lymphoma. Acta Ophthalmol. 2011;89(2):e149-54.

2 Bedaiwi K, Alfawaz AM, Mohammed SF, Maktabi AM, Alqahtani F, Alhazzaa S, et al. Intraocular T-cell lymphoma metastasizing from a primary adrenal T-cell lymphoma: case report. Ann Med Surg. 2020;60:646-50.

3 Eliassi-Rad B, Albert DM, Green WR. Frequency of ocular metastases in patients dying of cancer in eye bank populations. Br J Ophthalmol. 1996;80(2):125-8.

4 Alqurashi L, Alqahtani A. Ocular infiltration as the only relapse sign of atypical lymphoma under chemotherapy: a case report. Cureus. 2019;11(2):e4049.

5 Schocket LS, Syed NA, Fine SL. Primary adrenal lymphoma with choroidal metastases. Am J Ophthalmol. 2002; 134(5):775-6.

6 Rashidi A, Fisher SI. Primary adrenal lymphoma: a systematic review. Ann Hematol. 2013;92(12):1583-93.

7 Raoofziaee M, Yarmohamadi A, Ahmadnia H. Primary bilateral non-Hodgkin's lymphoma of the adrenal gland. Indian J Urol. 2018;34(4):300-2.

8 Pimentel M, Johnston JB, Allan DR, Greenberg H, Bernstein CN. Primary adrenal lymphoma associated with adrenal insufficiency: a distinct clinical entity. Leuk Lymphoma. 1997;24(3-4):363-7.

9 Salvatore JR, Ross RS. Primary bilateral adrenal lymphoma. Leuk Lymphoma. 1999;34(1-2):111-7.

10 Chamberlain MC, Glantz M, Groves MD, Wilson WH. Diagnostic tools for neoplastic meningitis: detecting disease, identifying patient risk, and determining benefit of treatment. Semin Oncol. 2009;36(4 Suppl 2):S35-45.

11 Tang LJ, Gu CL, Zhang P. Intraocular lymphoma. Int J Ophthalmol. 2017;10(8):1301-7.

12 Frenkel S, Hendler K, Siegal T, Shalom E, Pe'er J. Intravitreal methotrexate for treating vitreoretinal lymphoma: 10 years of experience. Br J Ophthalmol. 2008;92:383-8.

13 de Smet MD, Vancs VS, Kohler D, Solomon D, Chan CC. Intravitreal chemotherapy for the treatment of recurrent intraocular lymphoma. Br J Ophthalmol. 1999;83:448-51.

14 Kim E, Kim C, Lee J, Cho Y. A case of primary intraocular lymphoma treated by intravitreal methotrexate. Korean J Ophthalmol. 2009;23(3):210-4.

15 Sou R, Ohguro N, Maeda T, Saishin Y, Tano Y. Treatment of primary intraocular lymphoma with intravitreal methotrexate. Jpn J Ophthalmol. 2008;52(3):167-74.

16 Kitzmann AS, Pulido JS, Garrity JA, Witzig TE. Histologic findings in T-cell lymphoma infiltration of the optic nerve. Ophthalmology. 2008;115:e1-6.

17 Ohguro N, Hashida N, Tano Y. Effect of intravitreous rituximab injections in patients with recurrent ocular lesions associated with central nervous system lymphoma. Arch Ophthalmol. 2008;126:1002-3.

18 Itty S, Pulido JS. Rituximab for intraocular lymphoma. Retina. 2009;29:129-32. 